\title{
Solution of Non-Linear Poisson-Boltzmann Equation for Erythrocyte Membrane
}

\author{
Frederico A. O. Cruz, Fernando S. D. S. Vilhena, and Célia M. Cortez \\ Universidade do Estado do Rio de Janeiro \\ Departamento de Ciências Fisiológicas \\ Av. Prof Manuel de Abreu, 48 \\ 20550-170, Rio de Janeiro, RJ - Brazil
}

Received 25 October, 1999

\begin{abstract}
The space-dependent electric potential equations were determined by solution of non-linear PoissonBoltzmann equation, which decribes an erythrocyte membrane model which takes into account the charges of molecules associated with lipidic bilayer (glycocalyx molecules, outer side, and spectrin, inner side). It was assumed that on both sides of the membrane the charges are homogeneously distributed. Therefore, the potential was dependent on the z-coordinate only. Thus, we obtained the potential profile curve. The results are compared to previous ones obtained from more simple membrane model (Bioelectroch. Bioenerg. 1993, 32:305-315).
\end{abstract}

\section{Introduction}

It is well known that the passive transport of charged particles across biologic membranes is strongly affected by electrostatic forces and that the flux rates are dependent on intramembrane field forces [1-5]. But realistic values of membrane potential from experimental searches are obtained only for transmembrane potential (i.e., difference of potential between two points distant from outer and inner surfaces) [6]. The determination of potential profile, which describes the potential behaviour on all adjacent points along perpendicular axis to membrane, is performed based on theoretical models $[6,7,8]$. This is a way to estimate the potential in the vicinity of the membrane. However, informations from experimental searches are essential to formulate such mathematical models which allows the calculation of the membrane potential in function of space. Thus, several models have been suggested for biologic membrane and several electrostatic theories have been developped $[1,2,6,9]$. Some modern concepts, such as interionic correlations and surface conductivity, were already introduced within of the Gouy-Chapman theory, being its range of validity still discussed [10]. According to Cevc [6], the effects due to surface irregularities and to interfacial hydration decrease the significance of interionic correlation effects in several membranes.

In previous work [1], we associated some recent knowledge on membrane electrostatics with classical
Gouy-Chapmann model to describe erythrocyte membrane. For this model we performed a systematic study on the effects of ionic strength change on the electric potential profile. In the present work, we determine the potential profile for a complex membrane model, which takes into account electric charges of glycocalyx and spectrin layers. Donath and Patushenko [11] already have taken into account space charges of the glycocalyx for calculations of the outer surface potential. Heinrich et al [2] used space distributions of charges of glycocalyx, spectrin layer and hemoglobin for numerical calculation of potential profiles. Here, we determined the analytical solution for non-linear PoissonBoltzmann equation, which includes parameters due to fixed charges on glycocalyx and spectrin. The results are compared with previous results obtained for a simple model [1]. Analytical solution of the electric potential in neighboring of the membrane is of special importance for studies involving more complex mathematics, such as the study of mechanical stability of membrane by hydrodynamic analysis [12-16].

\section{Adopted model}

As already known, the erythrocyte membrane is constituted by a double bilayer of phospholipids, cholesterol and proteins [17], being asymmetric by distribution of phospholipids between the two lipid monolayers [18]. Molecules of phosphatidilserine and phos- 
phatidylethanolalanine are preferentially located in the inner monolayer and phosphatidylcholine and sphingomyelin are founded in the outer monolayer [19]. In the outer surface there is an additional layer rich in polysacharides, which are associated with the bilayer molecules, this is called the membrane glycocalyx layer. On the inner surface there is a protein trap that sup- ports the membrane, the spectrin [20]. In a solution of $\mathrm{NaCl}$ at $\mathrm{pH} 7.0$, erythrocytes exhibit a negative electrophoretic mobility. This is especially due to charged molecules of the glycocalyx layer [6]. In the same form, spectrin has a net negative charge at physiological $\mathrm{pH}$ [21]. In addition, all membranes are embedded in diffuse inorganic salt double layers [6].

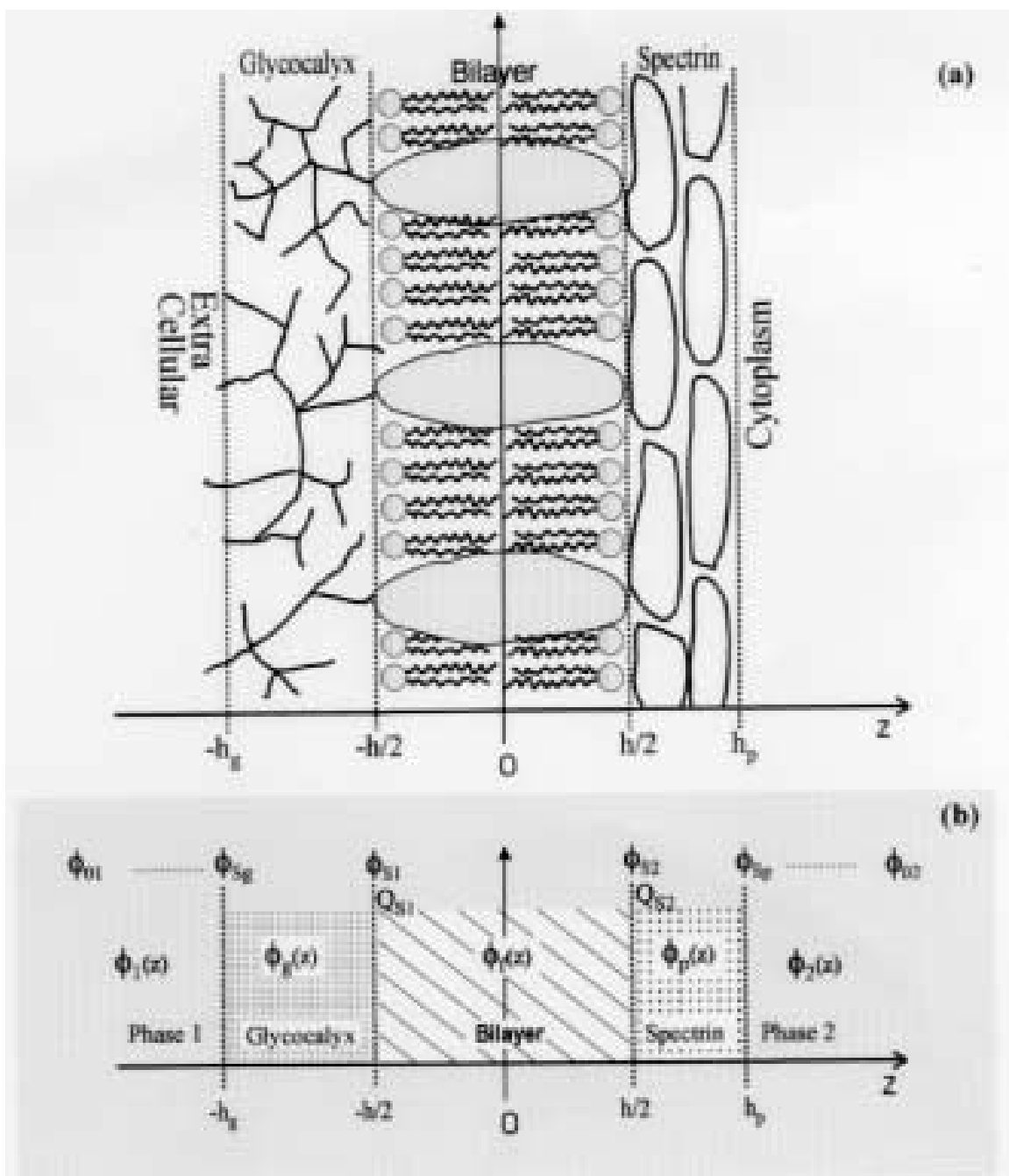

Figure 1. (a) Adopetd membrane model. The erythrocyte membrane is constituted by a lipidprotein bilayer, a outer layer rich in polysacharides, the glycocalyx, and a inner layer of spectrin. The bilayer surfaces located at positions $z= \pm h / 2$. The glicocalyx extends from $-h / 2$ to $-h_{g}$ and the spectrin layer extends from $h / 2$ to $h_{p}$. (b) Phases 1 and 2 correspond to extracellular medium and cytoplasm medium, respectively. $\phi_{1}(z)$ represents electric potential along $z$ axis into phase 1 , being $\phi_{01}$ the potential for $z \rightarrow-\infty$ and $\phi_{S g}$ the potential on $z=-h_{g}$ (on $S_{g}$ interface). In phase 2 , we have $\phi_{2}(z)$ as the electric potential along $z, \phi_{02}$ is the potential for $z \rightarrow \infty$ and $\phi_{S p}$ is the potential on $z=-h_{p}$ (on $S_{p}$ interface). Phases $g$ and $p$ correspond glycocalyx and spectrin layer, respectively, being $\phi_{g}(z)$ and $\phi_{p}(z)$ the electric potentials along $z$ axis in these phases. $S_{1}$ and $S_{2}$ are limit surface of bilayer and $\phi_{S 1}$ and $\phi_{S 2}$ are potentials on these interfaces. $Q_{S 1}$ and $Q_{S 2}$ are outer and inner surface charge density of bilayer.

Based on these knowledge, we adopted for our study the model shown in Fig. 1(a) and (b). The system is formed by five regions: extracellular bulk region (phase 1), glycocalyx layer (phase g), lipidic bilayer (phase f), spectrin layer (phase p) and cytoplasmatic region (phase 2). Within glycocalyx and spectrin layer there are space charge distributions denoted respectively by $\rho_{g}$ and $\rho_{p}$. The charge densities of these two later layers 
are considered almost constant in the $\mathrm{z}$ direction. The lipidic bilayer has dielectric constant $\epsilon_{f}$ and thickness $\mathrm{h}$ (extends from $\mathrm{z}=-\mathrm{h} / 2$ to $\mathrm{z}=\mathrm{h} / 2$ ), and it is limited by surfaces $S_{1}$ and $S_{2}$, as can be seen in Fig. 1(b). At these points the glycocalyx/bilayer and spectrin/bilayer interfaces are located, respectively. Similar to the Heinrich model [1], the inner surface charges are due only to the head group of phospholipids. So, there are electric charge distributions on $\mathrm{S} 1$ and $\mathrm{S} 2$ and their densities are denoted by $Q_{S 1}$ and $Q_{S 2}$ (Fig. 1(b)). The origin of the Cartesian coordinate axis is located at the bilayer centre. The electric potential depends only of the $\mathrm{z}$ coordinate, because charge distribution is considered constant along $\mathrm{x}$ and $\mathrm{y}$ axis. In aqueous phases, the electrochemical potentials, $\mu_{i}$, of each solute ion $\gamma$ are considered constant. The charge density $\rho$ in the bilayer film is taken as zero $\left(\rho_{i}(+)=\rho_{i(-)}=0\right) . S_{g}$ is the phase $1 /$ glycocalyx interface and $S_{p}$ is spectrin/phase 2 interface.

\section{Calculation of potential pro- file}

The Poisson equation applied for the five considered phases is

$$
\nabla^{2} \phi_{i}=-4 \pi \rho_{i} / \epsilon_{i} \quad i=1,2
$$

and it solution give us the electric potential $\phi(x, y, z)$ at any point in each one. The considered boundary are as follows (see Fig. 1 (b)):

- (I) When $z \rightarrow-\infty$ and $z \rightarrow \infty$, (a) the electric potential tends to limiting values $\phi_{01}$ and $\phi_{02}$, respectively, and (b) the corresponding ionic concentration tends to limiting values $\eta_{\gamma 01}$ and $\eta_{\gamma 02}$. In these regions one has the electroneutrality conditions: $\Sigma_{\gamma} Z_{\gamma} e \eta_{\gamma 01}=0$ and $\Sigma_{\gamma} Z_{\gamma} e \eta_{\gamma 02}=0$.

- (II) At $z=-h_{g}, z= \pm h / 2$ and $z=h_{p}$, the electric potential is continuous through the surfaces and takes the values $\phi_{S g}$ at the surfaces $S_{g}$ and $\phi_{S p}$ at surface $S_{p}, \phi_{S 1}$ and $\phi_{S 2}$ at $S_{2}$.

- (III) In these surfaces the condition of discontinuity of the electric displacement vector is valid.

Solution within phases 1 and 2. The potential equation for 1 and 2 phases already was calculated in previous paper [1]. In this regions we have only aqueous phase. Using the Boltzmann equilibrium, symmetric electrolytes and electroneutrality condition in both phases we obtained,

$$
\begin{gathered}
\phi_{i}=2 \ln \left(\frac{1+\chi_{i}}{1-\chi_{i}}\right)+\phi_{0 i} \\
\chi_{i}=\tanh \left(\frac{\beta}{4} \phi_{s o i}\right) \exp \left[k_{i}( \pm z+h / 2)\right]\left\{\begin{array}{l}
(+) \text { for } i=1 \\
(-) \text { for } i=2
\end{array}\right. \\
\phi_{s o i}=\phi_{s j}-\phi_{o i}
\end{gathered}
$$

$k_{i}$ is related to the of Debye constant, $\phi_{S j}$ the surface potential on $S_{j}(j=g, p), \phi_{o i}$ is the bulk potential in phase $i, \beta=Z e / K T, K$ is Boltzmann constant, $Z e$ is molar charge. The reference state is assumed to be stationary.

\section{Solution within glycocalyx and spectrin layers}

Within the Glycocalyx and spectrin layers the Poisson equation is non-linear due to addition of the term re- lated to fixed charges on these molecular nets [2].

$$
\nabla^{2} \phi_{j}(x, y, z)=-\frac{4 \pi}{\epsilon_{j}}\left(\rho_{j}+\rho_{f j}\right) \quad \text { for } j=g, p
$$

where $\rho_{j}$ is the electrolytic charge density and $\rho_{f j}$ refer to fixed charges into glycocalyx $\left(\rho_{f g}\right)$ or spectrin $\left(\rho_{f p}\right)$.

Using a Boltzmann distribution for the ions in aqueous phases and considering charge homogeneity on directions $x$ and $y$, we have for each type of ions: 


$$
\frac{d^{2}}{d z^{2}} \phi_{j}(z)=-\frac{4 \pi}{\epsilon_{j}}\left\{\rho_{f j}-2 Z_{0} e \eta_{0 j} \sinh \left[\frac{Z_{0} e}{K T}\left(\phi_{j}(z)-\phi_{s j}\right)\right]\right\}
$$

where $Z_{0} e$ is the molar electric charge $\eta_{0 j}$ the corresponding molar concentration of ions in solution within the phase $j$, and $\epsilon_{j}$ dielectric constant. In simplified form we have,

$$
\frac{d^{2}}{d z^{2}} \phi_{j}(z)=G_{j}+\zeta_{j} \sinh \left(\beta u_{j}\right)
$$

where

$$
\begin{gathered}
G_{j}=-\frac{4 \pi \rho_{f j}}{\epsilon_{j}} \\
\zeta_{j}=\frac{8 \pi Z_{0} \eta_{0 S} e}{\epsilon_{j}} \\
u_{j}=\phi_{j}(z)-\phi_{S j}
\end{gathered}
$$

The first order solution of equation 6 give us

$$
\frac{\partial \phi_{j}}{\partial z}=\left[G_{j} \phi_{j}(z)+\frac{2}{\beta} \cosh \left[\beta\left(\phi_{j}(z)-\phi_{s j}\right) \zeta_{j}\right]+C_{j}\right]^{1 / 2}
$$

Applying the boundary conditions II on $S_{g}$ e $S_{p}$, we have

$$
\frac{\partial \phi_{j}}{\partial z}=\left[2 G_{j} \phi_{j}(z)+\frac{2}{\beta} \cosh \left[\beta\left(\phi_{j}(z)-\phi_{s j}\right) \zeta_{j}\right]+2 G \phi_{s j}-\frac{2 \zeta_{j}}{\beta}+E_{s j}^{2}\right]^{1 / 2}
$$

where $E_{S j}=\partial \phi_{j} / \partial z$, at $S_{j}$.

Thus, we can write

$$
\int\left(g_{j} u_{j}+2 \sinh ^{2}\left(\beta u_{j} / 2\right)+\chi_{j}\right)^{-1 / 2} d u_{j}=k_{j} z
$$

being

$$
\chi_{j}=(1 / 4) \beta E_{S j}^{2} / \zeta_{i}
$$

$$
g_{i}=(1 / 2) G_{j} \beta / \zeta_{j}
$$

$$
k_{i}=2\left(\zeta_{i} / \beta\right)^{1 / 2}
$$

The solution of equation (13) is not trivial, hence simplifications was required. Using Taylor's expansion, we obtained that

$$
\left.\left[g_{j} u_{j}+2 \sinh ^{2} \beta u_{j} / 2\right)+\chi_{j}\right]^{-1 / 2}=a_{j}-(1 / 2) g_{j} a_{j}^{3} u_{j}+\left(-a_{j}^{2} \beta^{2}+3 / g_{j}^{2}\right) / 8 a_{j}^{2} u_{j}^{2}+\ldots .+
$$

which is a infinite series, where $a_{j}=\left(l / \chi_{j}\right)^{1 / 2}$.

Taking into account experimental data from literature for erythrocyte parameter values, and previous results [1], we studied the series terms. We found that, for $u_{j}<200 \mathrm{mV}$, the terms decrease significantly as the $u_{j}$-exponent increases. So the higher order terms do not contribute appreciably and the equation can be reduced to

$$
\int\left(a_{j}-b_{j}\right) u_{j} d u_{j}=k_{j} z
$$

where $b_{j}=(l / 2) g_{j} a_{j}^{3}$.
Solving the first order differential equation and considering electric potential continuity (condition II) at surfaces $S_{1}$ and $S_{2}$,

$$
\phi_{j}=\frac{-a_{j}+\left[a_{j}^{2}+2 b_{j}\left(\xi_{j}-k_{j}(z+h / 2)\right)\right]^{1 / 2}}{b j}+\phi_{S_{j}}
$$

being

$$
\xi_{j}=-a_{j}\left(\phi_{S i}-\phi_{S j}\right)+\left(1 / 2 b_{j}\right)\left(\phi_{S i}-\phi_{S j}\right)
$$

So, we can write: for glycocalyx region: 


$$
\phi_{1 g}=\frac{-a_{g}+\left[a_{g}^{2}+2 b_{g}\left(\xi_{g}-k_{g}(z+h / 2)\right)\right]^{1 / 2}}{b_{g}}+\phi_{S 1}
$$

where

$$
\xi_{g}=-a_{g}\left(\phi_{S 1}-\phi_{S g}\right)+\left(1 / 2 b_{g}\right)\left(\phi_{S 1}-\phi_{S g}\right)
$$

for spectrin region:

$$
\phi_{2 g}=\frac{-a_{p}-\left[a_{p}^{2}+2 b_{p}\left(\xi_{p}-k_{p}(z+h / 2)\right)\right]^{1 / 2}}{b_{p}}+\phi_{S 2}
$$

where

$$
\xi_{p}=-a_{p}\left(\phi_{S 2}-\phi_{S p}\right)+\left(1 / 2 b_{p}\right)\left(\phi_{S 2}-\phi_{S p}\right)
$$

Thus, from equations 18 and 19 we obtain, respectively,

$$
E_{S g}=k_{g} /\left[a_{g}^{2}+2 b_{g}\left(\xi_{g}-K_{g}\left(h_{g}-h / 2\right)\right)\right]^{1 / 2}
$$

and

$$
E_{S p}=k_{p} /\left[a_{p}^{2}+2 b_{p}\left(\xi_{p}-K_{p}\left(h_{p}-h / 2\right)\right)\right]^{1 / 2}
$$

\section{Solution within bilayer}

Considering that in lipidic bilayer charge density can be vanished, we have

$$
\nabla^{2} \phi_{f}(x, y, z)=O
$$

being the solution of a linear function family.

Taking into account the vector electric field displacement discontinuity (condition III) on the $S_{1}$ and $S_{2}$, and making some algebraic manipulations, we have:

$$
E_{f}=-\left(l / \epsilon_{f}\right)\left(\mp 4 \pi Q_{S i}+\frac{\epsilon_{j} k_{j}}{\left(a_{j}^{2}+2 b_{j} \xi_{i j}\right)^{(1 / 2)}}\right)
$$

being

$$
E_{f}=\left(\phi_{S 1}-\phi_{S 2}\right) / h
$$

$$
\xi_{s j}=\xi_{j}-K_{j}\left(h_{j}-h / 2\right)
$$

\section{Results}

Applying the referent values of the erythrocyte membrane (table 1) in results obtained here and using the interpolation method, we could trace the potential profile curve (Fig. 2). Fig. 3 shows the potential profile for the simple model based on the Gouy-Chapman theory of difuse layers, presented in anterior work [1].

Table 1. Numerical values os the parameters used of the calculations

\begin{tabular}{lccc}
\hline Parameter & Simbol & Value & Reference \\
\hline Inverse of Debye length & $(k) 1.34 \times 10^{9} \mathrm{~m}^{-1}$ & {$[1]$} & \\
Glycocalyx thickness & $\left(h_{g}\right)$ & $5.5 \mathrm{~nm}$ & {$[22]$} \\
Spectrin thickness & $\left(h_{p}\right)$ & $3.5 \mathrm{~nm}$ & {$[23]$} \\
Bilayer thickness & $(h)$ & $7.5 \mathrm{~nm}$ & {$[24]$} \\
Space charge density of glycocalyx & $\left(\rho_{g}\right)$ & $3.5 \times 10^{6} \mathrm{As} / \mathrm{m}^{3}$ & {$[25,26]$} \\
Space charge density of spectrin & $\left(\rho_{p}\right)$ & $-1.23 \times 10^{7} \mathrm{As} / \mathrm{m}^{3}$ & {$[21]$} \\
Inner Surface charge density & $\left(Q_{S 2}\right)$ & $-0.09 \mathrm{As} / \mathrm{m}^{2}$ & {$[2]$} \\
Aqueous dieletric constant & $\left(\epsilon_{i}, \epsilon_{j}\right)$ & 80 & \\
Bilayer dieletric constant & $\left(\epsilon_{f}\right)$ & 2 & \\
\hline
\end{tabular}

\section{Discussion and conclusions}

The inclusion of the volumetric charge distribution into Poisson's equation (equation 1) led us to a PoissonBoltzmann equation one with non linear characteristics and not trivial analytical solution. Thus, after search into possibilities we chose Taylor's series expansion to simplify its second integration (equation 13). However, we observed that the series (equation 15) was an infinit series, and did not converge for any potential value. Thus, it had required a detailed study to find the intervals where it became convergent, and if it was pos- sible into expected interval of potential values, taking into account numerical values of the involved potential equation parameters. So, we investigated the contribution of each series term and we verified convergence for numerical values of erythrocyte parameters. Indeed, the values of the terms decrease significantly with increasing of $u_{j}$-exponent, considering a potential interval between $0-200 \mathrm{mV}$, or $0 \leq\left(u_{i}-u_{j}\right) \leq 200 \mathrm{mV}$. Thus, it became possible to obtain a analytical result for potential, which can be easely used for posterior studies involving more complex calcules. The potential profile curve shown in the Fig. 2 was traced based on these 
obtained equations.

Using numerical integration, Heinrich et al [2] obtained the potential profile for their membrane model, which considered glycocalyx and spectrin charges hemoglobin charges. They studied the influence of ionic strength on this profile. Their results showed that, for all osmotic states studied, a negative inner surface potential $\left(S_{2}\right)$ of more than $60 \mathrm{mV}$ was calculated. For a ionic strength of $145 \mathrm{mM}$ the potential on the $S_{2}$ was about $-75 \mathrm{mV}$, and on $S_{1}$ (outer bilayer surface) was about $-10 \mathrm{mV}$. They also shown that at a decrease of ionic strength causes a increase of negativity of potential on $S_{2}$. Our potential profile (Fig. 2), which was obtained from analitical solution of Poisson-Boltzmann equation, presents the same shape than those shown by Heinrich et al [2]. We considered in our calculation a ionic strength of $172 \mathrm{mM}$.

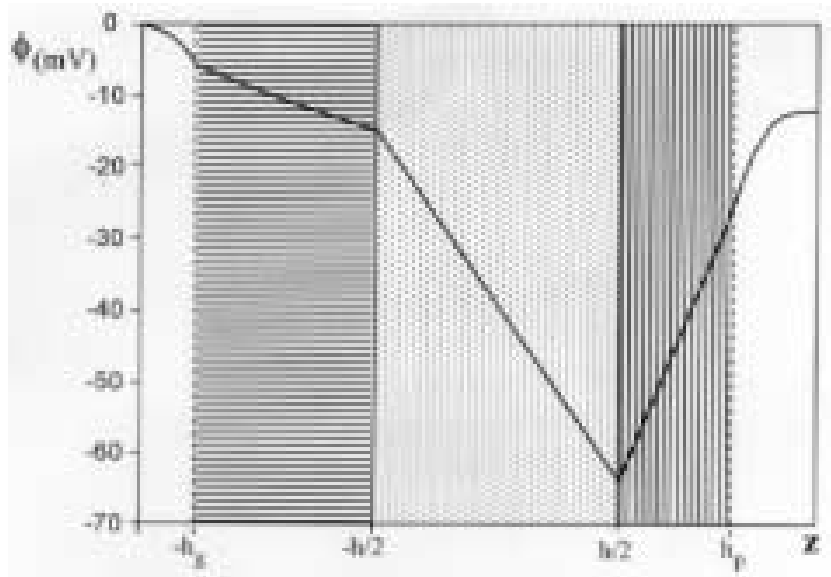

Figure 2. Electric Potential Profile of the erythrocyte membrane calculated by use of the potential equations from analytical solution of nonlinear Poisson-Boltzmann equation. Numerical values adopted are shown in table 1. (Ionic strength $=172 \mathrm{mM}$ )

In a previous paper [1], we adopted a simple model, which was based on the Gouy-Chapman theory of diffuse layers, in which space distributions of fixed charge of glycocalyx and spectrin layers was neglected. Comparing the curve of Fig. 2 with that of previous results (Fig. 3), we observed that the profile suffers some change when we took into account these additional charges. Within layers where there are fixed charges, the potential varies less pronouncedly than when these space distribution of charges are neglected. In this case (Fig. 3), the observed potential varies exponentially in both two sides, and the linear part of the inner curve presents a pronounced inclination. As can be seen in Fig. 2, the inclination of potential profile in the neighboring of the bilayer is smaller than that shown in Fig. 3 in the same place. In the simple model [1], we adopted a distance of $10 \mathrm{~nm}$ between the two parallel planes which limit the membrane region. So, the outer surface was located approximately at half the distance between the bilayer surface and the more extreme point of glycocalyx layer. All charges belonging to this membrane side (bilayer surface and neighbouring fixed charges) were taken into account as surface charge. Thus, in that case, we circumvented space charges by treating them as surface charges becoming trivial the solution of the Poisson-Boltzmann equation by analytical means. By confront of these two figures, it can be observed the importance of glycocalyx charges on potential within the outer membrane surface, as well as the potential within spectrin layer at the inner surface is mainly determined by the charges in this region.

Applying the analytical solution of the electric potential of the membrane in the hydrodynamic analysis, we can study the influence of these charges on the mechanical stability of the membrane. As can be seen in Fig. 2, in spectrin region there is an important electric field, and its effect on transport phenomena through membrane may be imperative, as well as on regulation of other membrane functions.

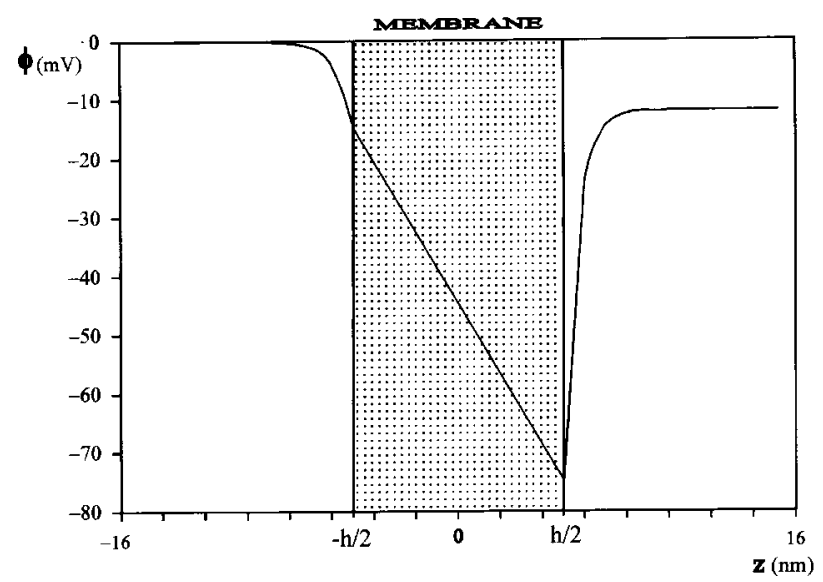

Figure 3. Electric Potential Profile of the erythrocyte membrane calculated by use of the potential equations from analytical solution of the linear Poisson-Boltzmann equation. $Q_{S 1}=1.41 \times 10^{-3}$ A.s $/ \mathrm{m}^{2}, Q_{S 2}=-9 \times 10^{-2}$ A.s $/ \mathrm{m}^{2}, h=10$ $\mathrm{nm}$. (Ionic strength $=172 \mathrm{mM}$ ).

\section{References}

[1] C. Cortez Maghelly and P.M. Bisch. Bioelectroch. and Bioenerg 32, 305 (1993).

[2] R. Heinrich, M. Gaestel and R. Glaser. J. Theor. Biol. 96, 211 (1982).

[3] S.J. Kennedy. J. Membrane Biol. 42, 265 (1978).

[4] R. Glaser, I. Bernhardt and E. Donath. J. Electroanl. Chem. 116, 281 (1980).

[5] G. Schwarz. J. membrane Biol. 43, 127 (1978).

[6] G. Cevc. Biochim. Biophys. Acta 1031, 311 (1990). 
[7] J. Baker, W.T. Coakley and D. Gallez. Eur. Biophys. 22, 53 (1993).

[8] H.P. Schnebll, C. Roeder and L. Tarcsay. Experimental Cell Research 98, 273 (1976).

[9] C.C. Wang, L.J. Bruner. Biophys. J. 24, 749 (1978).

[10] E. Donath and A. Voigt. Biophys. J. 49, 493 (1989).

[11] E. Donath and V. Pastushenko. J. Electroanal. Chem. 104, 543 (1979).

[12] M. Prevost, P.M. Bisch and A. Sanfeld. J. Colloid Interface Sci. 88, 353 (1982).

[13] P.M. Bisch, H. Wendel and D. Gallez. J. Colloid Interface Sci. 92, 105 (1983).

[14] W.T. Coakley and D. Gallez. Interfacial Stability. In: Biophysics of the Cell Surface (Glaser, R. \& Gingell, D., eds), p.287. Berlin (1990) Springer Verlag.

[15] C. Cortez Maghelly and P.M. Bisch. J. Theor. Biology 176, 325 (1995).

[16] C. Cortez-Maghelly and P.M. Bisch. Braz. J. Phys. 26, 604 (1996).

[17] E.M. Manevich, K.M. Labin and A.I. Archakov. Biochim. Biophys. Acta, 815, 455 (1985).
[18] P. Gascard, D. Tran, M. Sauvage, J.C. Sulpice, K. Fukami, T. Takenawa, M. Claret and F. Girand. Biophys. Chem. 1069, 27 (1991).

[19] P.J. Raval and D. Allan. Biochim. Biophys. Acta. 722, 192 (1982).

[20] G.L Nicolson, V.T. Marchesi and S.J. Singer J. Cell. Biol. 51, 265 (1971).

[21] A. Elgsaeter, D.M. Shotton and D. Branton. Nature 307, 378 (1984).

[22] E. Donath and D. Lerche. J. electroanal. Chem. 116, 41 (1980).

[23] M. Blank, R.G. King, L. Soo, Re. Abbott and S. Chim. J. Coll. Interface Sci. 69, 67 (1979).

[24] D.S. Parson. Biological Membranes. Oxford (1975) clarendon Press.

[25] G.M.W. Cook, D.H. Heard and G.V.F. Seaman. Nature. 191, 4449 (1961).

[26] R. Schauer, A.P. Cornfield, M. Wember and D. Danon, Physiol. Chem. 356, 1727 (1975). 